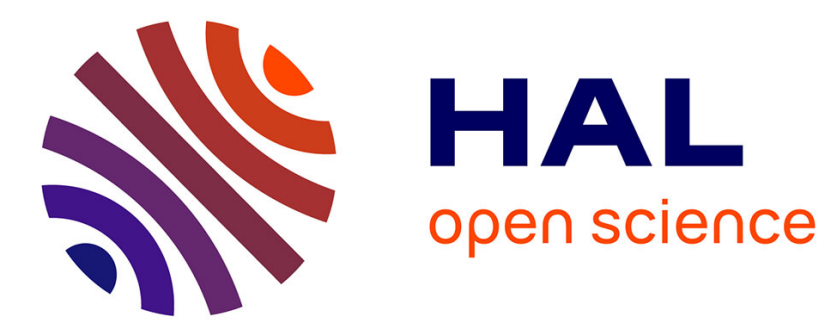

\title{
Plasma créé en impulsion par une onde de plasma
}

E. Bloyet, P. Leprince, J. Marec, M. Moisan

\section{To cite this version:}

E. Bloyet, P. Leprince, J. Marec, M. Moisan. Plasma créé en impulsion par une onde de plasma. Revue de Physique Appliquée, 1977, 12 (10), pp.1719-1722. 10.1051/rphysap:0197700120100171900 . jpa-00244392

\section{HAL Id: jpa-00244392 https://hal.science/jpa-00244392}

Submitted on 1 Jan 1977

HAL is a multi-disciplinary open access archive for the deposit and dissemination of scientific research documents, whether they are published or not. The documents may come from teaching and research institutions in France or abroad, or from public or private research centers.
L'archive ouverte pluridisciplinaire HAL, est destinée au dépôt et à la diffusion de documents scientifiques de niveau recherche, publiés ou non, émanant des établissements d'enseignement et de recherche français ou étrangers, des laboratoires publics ou privés. 


\title{
PLASMA CRÉÉ EN IMPULSION PAR UNE ONDE DE PLASMA (*)
}

\author{
E. BLOYET, P. LEPRINCE, J. MAREC \\ Laboratoire de Physique des Plasmas (**), Bâtiment 212, Université Paris XI, Centre d'Orsay, \\ 91405 Orsay, France
}

et

M. MOISAN

Laboratoire de Physique des Plasmas, Université de Montréal, Canada

(Reçu le 30 décembre 1976, révisé le 18 avril 1977, accepté le 23 juin 1977).

\begin{abstract}
Résumé. - Lorsqu'un plasma est créé en impulsion par une onde de plasma, l'ionisation n'est pas simultanée en tous points de l'enceinte; on observe la présence d'un front d'ionisation et d'un front d'onde dont les vitesses sont égales et très petites par rapport à la vitesse de groupe de l'onde. Des mesures du gradient du carré du champ électrique le long de la colonne ont montré que la vitesse est due à une accélération des électrons par ce gradient pendant un temps de l'ordre du temps entre deux collisions.
\end{abstract}

\begin{abstract}
If a pulsed plasma is created by a plasma wave, the ionization does not occur simultaneously everywhere in the discharge tube. An ionization front and a wave one are observed, their velocities are equal and lower than the group velocity of the wave. The front velocity is due to an electrons acceleration by a gradient of the electrical field located at the end of the plasma.
\end{abstract}

1. Introduction. - Lorsqu'on étudie les processus de création d'un plasma par un champ de haute fréquence on suppose que ce champ $E$ est appliqué simultanément en tous points de la zone où l'on crée le plasma, il est alors possible de définir un temps d'ionisation qui dépend de la nature du gaz et du champ $E$. Cette description est valable pour toutes les structures usuelles (tube dans un guide ou dans une cavité par exemple). Par contre dans le cas où le plasma est créé par une onde il faut tenir compte de la vitesse $v_{\mathrm{f}}$ du front d'onde. Si l'onde peut se propager sans la présence du plasma (mode de guide [1]) la vitesse $v_{\mathrm{f}}$ est égale à la vitesse de groupe $v_{\mathrm{g}}$ de l'onde; le temps de propagation est en général petit devant le temps d'ionisation et par suite l'hypothèse précédente est valable. Si l'onde ne peut se propager qu'en présence de plasma (mode plasma [1]), la vitesse du front d'onde dépendra du temps d'ionisation et l'on observera la présence d'un front d'ionisation.

Dans cet article, nous avons étudié le mécanisme de création du plasma dans le cas où l'on excite une onde de plasma (onde de surface) en nous intéressant spécialement à la vitesse du front d'ionisation.

$\left({ }^{*}\right)$ Communication présentée au Congrès National de Physique des Plasmas, 6-10 décembre 1976.

$\left({ }^{* *}\right)$ Laboratoire associé au C.N.R.S.
Dans le cas d'un fonctionnement en continu, on observe que :

- la densité est telle que la fréquence d'excitation $f_{\mathrm{o}}$ est toujours inférieure à la fréquence plasma $f_{\mathrm{p}}$

$$
f_{\mathrm{o}} \leqslant \frac{f_{\mathrm{p}}}{\left(1+\varepsilon_{\mathrm{v}}\right)^{1 / 2}}
$$

où $\varepsilon_{\mathrm{v}}$ est la constante diélectrique de l'enveloppe;

- l'onde qui se propage le long de la colonne de plasma a bien les caractéristiques de l'onde de surface [2];

- le champ $E$ décroit à partir de la source et son atténuation est donnée par [3]

$$
\alpha=\frac{\langle v\rangle}{2} \cdot v_{\mathrm{g}}
$$

où $\langle v\rangle$ est la fréquence de collision.

Dans le cas d'un fonctionnement en impulsion, les propriétés du plasma sont identiques; on observe :

- la propagation de l'onde tant que la densité est supérieure à un certain seuil et donc une réflexion de l'onde au bout du plasma;

- la présence d'ondes stationnaires qui permettent (en mesurant la longueur d'onde) de vérifier que l'onde excitée est bien l'onde de surface.

2. Résultats expérimentaux. - Le montage expérimental est indiqué sur la figure 1. La source de 


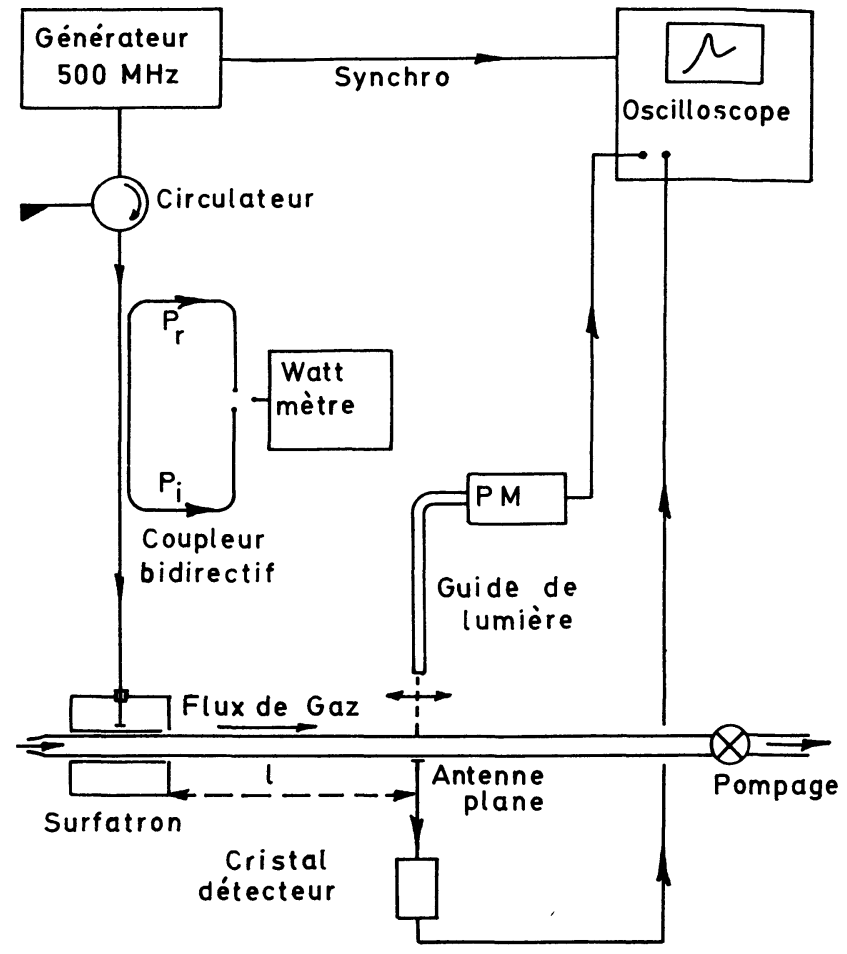

FIG. 1. - Montage expérimental.

[Experimental design.]

plasma est un surfatron [4] fonctionnant en impulsion $\left(f_{\mathrm{o}}=500 \mathrm{MHz}\right.$, largeur de l'impulsion $=500 \mu \mathrm{s}$, fréquence de répétition 750 à $1250 \mathrm{~Hz}$; puissance crête 30 à $100 \mathrm{~W}$ ). La lumière du plasma et le champ électrique le long de la décharge sont recueillis par un guide de lumière et une antenne placés perpendiculairement à l'axe du tube et mobiles le long de cet axe. Il est ainsi possible de mesurer la vitesse du front d'ionisation (lumière) et celle du front de l'onde (antenne).

La figure 2 montre un exemple d'impulsions de lumière et H.F. détectées à trois distances différentes $l$ (cf. Fig. 1) du gap, l'origine des temps étant l'instant d'application de l'impulsion H.F. sur le coupleur. Sur cette figure, on peut faire les observations suivantes : l'impulsion H.F. et l'impulsion de lumière sont détectées avec un temps de retard $t_{\mathrm{r}}$ qui croit avec $l$. En fait, l'impulsion de lumière apparaît avec un très faible retard sur l'impulsion H.F. ce qui est normal car il faut un minimum de densité pour qu'il $\mathrm{y}$ ait de la lumière. La simultanéité de la propagation des deux fronts s'explique en rappelant que l'onde ne peut se propager tant que la densité électronique $n_{\mathrm{e}}$ reste inférieure à une valeur seuil $n_{\mathrm{s}}$ définie par

$$
f_{\mathrm{ps}}=\left(\frac{n_{\mathrm{s}} e^{2}}{2 \pi m \varepsilon_{\mathrm{o}}}\right)^{1 / 2}=f_{\mathrm{o}}\left(1+\varepsilon_{\mathrm{v}}\right)^{1 / 2}
$$

Or, à un instant donné, au bout du plasma, il y a une chute rapide de la densité (sur environ $1 \mathrm{~cm}$ ). Ce gradient de densité réfléchit l'onde car la vitesse de propagation $\mathrm{du}$ front est très inférieure à la vitesse de groupe $v_{\mathrm{g}}$ de l'onde, ces réflexions donnent naissance à un système d'ondes stationnaires qui explique les ondulations du signal H.F. observées sur la figure 2. Il y a donc un fort gradient de $E^{2}$. De ce temps d'apparition de la lumière (ou du signal H.F.), on peut déduire la vitesse du front d'ionisation. Les résultats portés sur la figure 3 montrent les variations de la

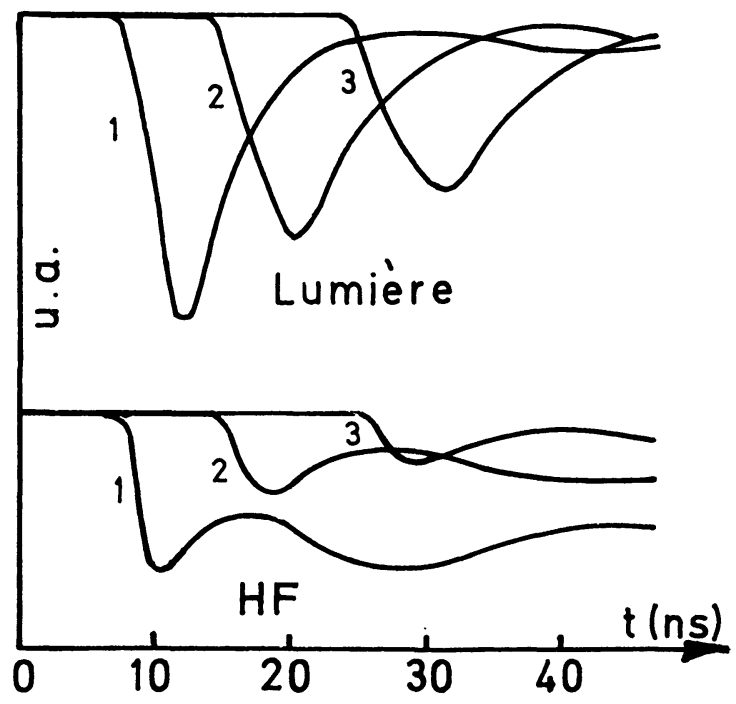

Fig. 2. - Impulsions de lumière et H.F. détectées pour trois valeurs de $l: l_{1}=16 \mathrm{~cm} ; l_{2}=26 \mathrm{~cm} ; l_{3}=36 \mathrm{~cm}$. Argon : $p=1$ torr $; \varnothing_{\text {plasma }}=9 \mathrm{~mm} ; P_{\text {inc }}=50 \mathrm{~W}$ crête.

[Light and R.F. pulses : $l_{1}=16 \mathrm{~cm} ; l_{2}=26 \mathrm{~cm} ; l_{3}=36 \mathrm{~cm}$. Argon: $p=1$ torr $; \varnothing_{\text {plasma }}=9 \mathrm{~mm} ; P_{\text {in }} \sim 50 \mathrm{~W}$ peak to peak.]

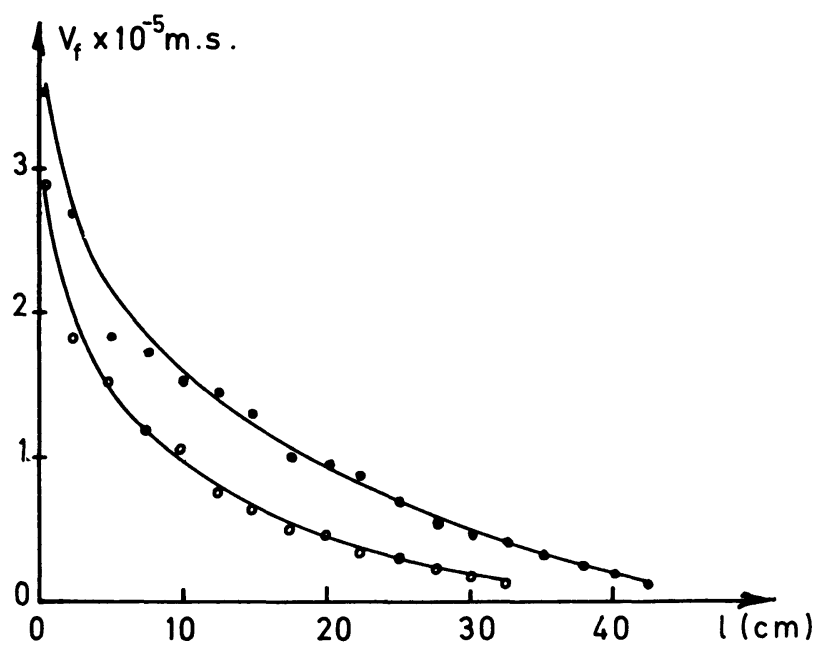

Fig. 3. - Vitesse du front le long de la décharge. Argon : $\varnothing_{\text {plasma }}=9 \mathrm{~mm} . P_{\text {inc }}=50 \mathrm{~W}$. Pression : $\bullet 0,15$ torr, $\bigcirc 0,25$ torr.

[Front velocity along the discharge. Argon : $\varnothing_{\text {plasma }}=9 \mathrm{~mm}$. $P_{\text {in }}=50 \mathrm{~W}$ peak to peak. Pressure $=0.15$ torr $\bullet, 0.25$ torr O.] 
vitesse instantanée $v_{\mathrm{f}}$ du front (mesurée par le retard du maximum de champ pour des positions voisines de l'antenne) pour deux pressions différentes. Les deux courbes indiquent que $v_{\mathrm{f}}$ dépend de la pression et décroit avec le champ électrique de l'onde (on sait en effet que dans ce type de plasma le champ $E$ décroit d'une manière continue depuis l'excitateur [4]). Néanmoins, la vitesse du front d'ionisation ne peut pas s'expliquer par le mouvement des électrons dans le champ oscillant $\left(v_{\mathrm{f}}>v_{\mathrm{osc}}=\frac{e E}{m \omega}\right)$. Par contre le gradient de champ à l'extrémité du plasma provoque une éjection des électrons du plasma; en effet ils sont soumis à une force

$$
F=\frac{e^{2}}{m \omega^{2}} \nabla E^{2}
$$

qui accélère les électrons dont la vitesse, dirigée suivant l'axe du tube, après un temps $\tau$ est donnée par

$$
v_{\mathrm{o}}=\frac{e^{2}}{m^{2} \omega^{2}} \tau \cdot \nabla E^{2}
$$

Dans notre expérience, ce temps est déterminé par le temps $\tau_{c}$ entre deux collisions qui est beaucoup plus petit que celui pendant lequel l'électron se trouve dans le gradient de champ. La vitesse moyenne des électrons

$$
\bar{v}_{\mathrm{e}}=\frac{1}{2} \frac{e^{2}}{m^{2} \omega^{2}} \tau_{\mathrm{c}}\left|\nabla E^{2}\right|
$$

peut sans doute expliquer la vitesse du front d'ionisation et c'est ce que nous avons essayé de vérifier expérimentalement en étudiant la loi de variation de $v_{\mathrm{e}}$ en fonction du $\nabla E^{2}$ et de la pression.

La figure 4 donne, pour deux pressions, $v_{\mathrm{f}}$ en fonction du $\left|\nabla E^{2}\right|$ obtenu en mesurant expérimentalement $\Delta E^{2} / \Delta t$ (Fig. 3) et en utilisant l'équation

$$
\frac{\mathrm{d} E^{2}}{\mathrm{~d} x}=\frac{1}{v_{\mathrm{f}}} \cdot \frac{\mathrm{d} E^{2}}{\mathrm{~d} t}
$$

On remarquera tout d'abord que $\left|\nabla E^{2}\right|$ est donné en unités arbitraires car il n'est pas possible d'avoir une mesure absolue du champ électrique, on sait seulement que le signal H.F. détecté est proportionnel à $E^{2}$. Ceci étant, on peut constater sur cette figure que la loi de proportionnalité entre $v_{\mathrm{f}}$ et $\left|\nabla E^{2}\right|$ est vérifiée de façon satisfaisante (à environ $15 \%$ près) compte tenu des erreurs expérimentales liées à la mesure du gradient de $E^{2}$ (près du gap d'excitation la mesure de $E^{2}$ est perturbée par le champ de rayonnement proche de surfatron et loin du gap, la détermination du temps de montée de l'impulsion H.F. est rendue imprécise du fait de l'amortissement de l'onde).

La figure 5 montre l'influence des collisions (on suppose que $\tau_{\mathrm{c}}$ est proportionnel à $1 / p$ ) sur la vitesse $v_{\mathrm{f}}$ ou plus exactement sur le rapport $v_{\mathrm{f}} /\left|\nabla E^{2}\right|$; la précision des mesures est de l'ordre de $25 \%$.

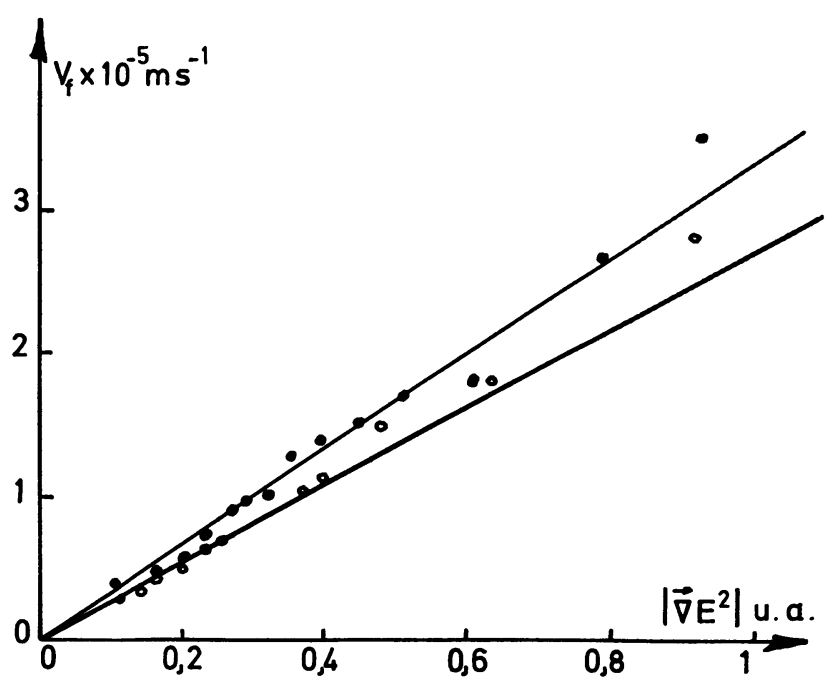

Fig. 4. - Vitesse du front en fonction de $\nabla \mathbf{E}^{2}$ mêmes conditions que figure 3.

[Front velocity versus $\nabla E^{2}$ same conditions as in figure 3.]

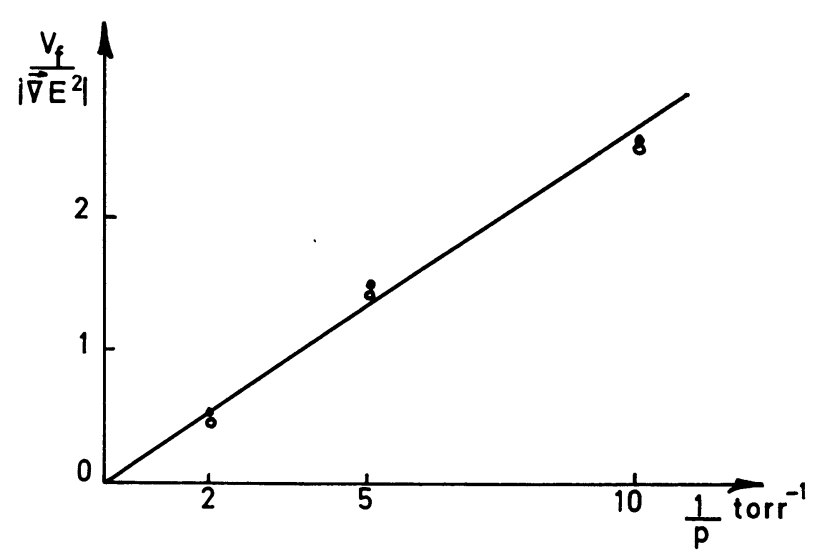

FIG. 5. - Dépendance de la vitesse du front avec la pression. Argon : $\varnothing_{\text {plasma }}=9 \mathrm{~mm} . P_{\text {inc }}=50 \mathrm{~W}, \bullet l=15 \mathrm{~cm}, \circ l=25 \mathrm{~cm}$.

[Front velocity dependence with pressure. Argon: $\varnothing_{\text {plasma }}=9 \mathrm{~mm}$. $P_{\text {in }} \sim 50 \mathrm{~W}$ peak to peak, $\bullet l=15 . \mathrm{cm}, \circ l=25 \mathrm{~cm}$.]

3. Discussion et conclusion. - Ces deux courbes montrent que, dans le domaine des paramètres de l'expérience $\left(P_{\mathrm{HF}}<100 \mathrm{~W}, 0,1<p<0,6\right.$ torr $)$ la vitesse $d u$ front d'ionisation est donnée par la vitesse d'éjection des électrons par le gradient de champ.

On peut aussi remarquer, du point de vue des ordres de grandeur que la vitesse du front d'ionisation $v_{\mathrm{f}}$ est très inférieure à la vitesse de groupe $v_{\mathrm{g}}$ de l'onde. On a en effet pour $v_{\mathrm{f}}$ des valeurs voisines de $10^{5} \mathrm{~m} / \mathrm{s}$ alors que dans nos conditions de travail $\left(f_{\mathrm{pe}} \sim 2 \mathrm{GHz}\right)$ $v_{\mathrm{g}}$ est voisine de $10^{7} \mathrm{~m} / \mathrm{s}$ (détermination par le calcul 
à partir de la courbe de dispersion du mode $m=0$ du surfatron et d'une mesure de longueur d'onde dans le plasma). Nous avons déterminé expérimentalement la vitesse $v_{\mathrm{f}}$ mais celle-ci peut également être calculée à partir du flux de la puissance transportée par l'onde de surface en faisant un bilan d'énergie simple. Nous allons calculer cette vitesse et la comparer à nos mesures. Rappelons que, si $P s$ (où $s=\pi a^{2}$ est la section du plasma) est le flux de la puissance transportée par l'onde à une distance $l$ du gap d'excitation, le champ électrique moyen de l'onde à cette distance est donné par la relation :

$$
E^{2}=\frac{4 P}{\pi a^{2} \varepsilon_{\mathrm{o}} v_{\mathrm{g}}\left|1-\frac{\omega^{2}}{\omega^{2}}\right|}
$$

avec $\omega_{\mathrm{p}}=2 \pi f_{\mathrm{p}}$ et $\omega=2 \pi f_{\mathrm{o}}$. Du champ ainsi calculé, on peut déduire la vitesse d'éjection des électrons en utilisant l'équation (6). On trouve pour une puissance de $50 \mathrm{~W}\left(E \simeq 0,5 \times 10^{5} \mathrm{~V} / \mathrm{m}\right)$ avec une fréquence de collision $10^{8} \mathrm{~Hz}(p \simeq 0,25$ torr)

$$
\bar{v}_{\mathrm{e}} \simeq 4,5 \times 10^{5} \mathrm{~m} / \mathrm{s}
$$

tandis qu'expérimentalement près du gap (donc dans une zone où la puissance transportée est voisine de $50 \mathrm{~W}$ )

$$
v_{\mathrm{f}} \simeq 3 \times 10^{5} \mathrm{~m} / \mathrm{s}
$$

Il n'y a pas un accord parfait entre la théorie simple que nous avons faite et les mesures mais néanmoins l'ordre de grandeur est respecté ce qui nous paraît excellent compte tenu de la précision des mesures. De plus, la proportionnalité de $v_{\mathrm{f}}$ avec $\left|\nabla E^{2}\right|$ indique bien que la vitesse de front d'ionisation est due à l'éjection des particules par le gradient de champ provoqué par la réflexion de l'onde sur le gradient de densité.

\section{Bibliographie}

[1] Bloyet E., Leprince P. et Milleon H., J. Physique 34 (1973) 13.

[2] Trivelpiece A. W. et Gould R. W., J. Appl. Phys. 30 (1959) 1784.

[3] Zahrzewski Z., Moisan M., Glaude V.M.M., Beaudry C. et LePRINCE P., à paraître dans Plasma Phys.

[4] Moisan M., Beaudry C. et Leprince P., IEEE Trans. Plasma Sci. PS 3 (1975) 35. 